\title{
A Photochemical Equilibrium Model for Ionospheric Conductivity
}

\author{
C. E. RASMUSSEN AND R. W. SchUNK \\ Center for Atmospheric and Space Sciences, Utah State University, Logan \\ V. B. WICKWAR \\ SRI International, Menlo Park, California
}

\begin{abstract}
A photochemical equilibrium model of the high-latitude ionosphere has been developed. This model provides densities of the ionospheric constituents, $\mathrm{N}_{2}^{+}, \mathrm{O}_{2}^{+}, \mathrm{O}^{+}$, and $\mathrm{NO}^{+}$, from $85 \mathrm{~km}$ to approximately 220 $\mathrm{km}$. These densities are then used to calculate Pedersen and Hall conductivities. A comparison of the model results with Arecibo and Chatanika radar observations was made, covering periods of solar minimum and solar maximum. The comparison showed the model to predict ionospheric densities to within $50 \%$ and conductivities to within $40 \%$ in the illuminated portion of the ionosphere. In regions of electron precipitation, the model showed good agreement with measurements. Results of this study indicate the following: (1) Ionospheric conductivity increases by a factor of $\sim 1.6$ from solar minimum to solar maximum conditions; (2) the portion of the ionosphere above $170 \mathrm{~km}$ can contribute as much as $40 \%$ during daylight and 80\% during nighttime to the total height-integrated Pedersen conductivity; (3) the ratio of the heightintegrated Hall to Pedersen conductivities is approximately 1.1-1.3 for sunlit conditions; this is appreciably lower than the value of 2 found in previous studies; (4) these and other factors indicate that, under certain conditions, the height-integrated Pedersen conductivity may be as much as 2-3 times larger than previously reported.
\end{abstract}

\section{INTRODUCTION}

The conductivity of the ionosphere plays a critical role in various coupling processes important to geophysics. One of the most important is the part it plays in magnetosphere-ionosphere coupling, where magnetospheric Birkeland currents feed the ionospheric system of electric fields, currents, and conductivity. Of equal importance is the coupling of the ionosphere to the thermosphere. Here, ionospheric conductivity plays a role in the transfer of energy between the two systems via Joule heating, and it also links the thermospheric wind system to ionospheric electric fields.

The importance of conductivity to geophysical processes is evident in the number of models which require ionospheric conductivity as an input. For instance, a model of ionospheric conductivity is needed by magnetospheric and thermospheric models [Harel et al., 1981; Roble et al., 1982; St.-Maurice and Schunk, 1981] and is needed for studies of the dynamics of magnetosphere-ionosphere coupling [Lysak and Dum, 1983]. A model of Hall and Pedersen conductivities is also needed in the inversion of magnetometer data to obtain ionospheric electric fields [Kamide et al., 1981] and in other ionospheric convection models [e.g., Kamide and Matsushita, 1979; Rasmussen and Schunk, 1987].

Several studies of Hall and Pedersen conductivities have been made based on radar and satellite measurements. Of these, two studies examined the contribution of solar illumination to conductivity, and empirical models based on the solar zenith angle were developed [Mehta, 1978; Vickrey et al., 1981]. Although small, the conductance of the nocturnal atmosphere is nonzero and has been examined using Arecibo radar measurements [Rowe and Mathews, 1973; Harper and Walker, 1977]. Others have used the Chatanika radar to study the auroral contribution

\section{Copyright 1988 by the American Geophysical Union.}

Paper number 7 A9274.

0148-0227/88/007A-9274\$05.00 to conductivity [Brekke et al., 1974; Horwitz et al., 1978; Vickrey et al., 1981]. Satellite measurements of electron precipitation have been used in conjunction with the Rees [1963] model for auroral production to develop empirical models of auroralzone conductance, averaged according to the $K \boldsymbol{p}$ [Wallis and Budzinski, 1981] and $A E$ [Spiro et al., 1982] indices (see also Fuller-Rowell and Evans [1987]). In a recent study, auroral images were used to infer instantaneous conductivities [Kamide et al., 1986]. Finally, the subject of high-latitude conductivity has been reviewed by Reiff [1983].

For this study we developed a theoretical model of ionospheric conductance. This model calculates ion densities in the $E$ and lower $F$ regions by numerically solving the chemical reaction of four ions, $\mathrm{N}_{2}^{+}, \mathrm{O}_{2}^{+}, \mathrm{O}^{+}$, and $\mathrm{NO}^{+}$. One major assumption of the model is that this region of the ionosphere is in photochemical equilibrium. The ion densities are then used to calculate Hall and Pedersen conductivities.

One of the advantages of a theoretical model is that the effects of parameters, such as the solar flux, can be easily studied. Since this is one of the reasons this study has been undertaken, we have examined the effects of the solar zenith angle, the solar $10.7-\mathrm{cm}$ flux, and the energy of electron precipitation on ionospheric conductivity. Comparisons of electron densities and conductance with radar measurements have also been made.

\section{CONDUCTIVITY MODEL}

In the ionosphere, conduction perpendicular to the ambient magnetic field is provided by collisions between charged species (both electrons and ions) and the neutral gas. In this section a mathematical model of ionospheric conductivity is described. Briefly, the model consists of solving simplified versions of the continuity equations for the ionic species, after which the conductivity tensor is obtained from the momentum equations. Only an outline of the model is given here, since more details of the implementation of the computer model are given in the appendix. 
TABLE 1. Ion Chemistry

\begin{tabular}{|c|c|c|c|}
\hline \multicolumn{4}{|c|}{ Reaction } \\
\hline $\mathbf{R} \mathbf{1}$ & $\mathbf{N}_{2}+h \nu$ & $\rightarrow$ & $\mathbf{N}_{2}^{+}+e$ \\
\hline $\mathbf{R 2}$ & $\mathrm{O}_{2}+h \nu$ & $\rightarrow$ & $\mathrm{O}_{2}^{+}+e$ \\
\hline $\mathbf{R 3}$ & $O+h \nu$ & $\rightarrow$ & $\mathrm{O}^{+}+e$ \\
\hline $\mathbf{R 4}$ & $\mathrm{N}_{2}^{+}+e$ & $\rightarrow$ & $\mathbf{N}+\mathbf{N}$ \\
\hline R5 & $\mathrm{O}_{2}^{+}+e$ & $\rightarrow$ & $0+0$ \\
\hline R6 & $\mathrm{NO}^{+}+e$ & $\rightarrow$ & $\mathbf{N}+\mathbf{O}$ \\
\hline $\mathbf{R 7}$ & $\mathbf{N}_{2}^{+}+\mathbf{O}_{2}$ & $\rightarrow$ & $\mathbf{O}_{2}^{+}+\mathbf{N}_{2}$ \\
\hline R8 & $\mathrm{N}_{2}^{+}+\mathrm{O}$ & $\rightarrow$ & $\mathrm{NO}^{+}+\mathbf{N}$ \\
\hline R9 & $\mathbf{N}_{2}^{+}+\mathbf{O}$ & $\rightarrow$ & $\mathrm{O}^{+}+\mathrm{N}_{2}$ \\
\hline R10 & $\mathrm{N}_{2}^{+}+\mathrm{NO}$ & $\rightarrow$ & $\mathrm{NO}^{+}+\mathrm{N}_{2}$ \\
\hline R11 & $\mathbf{O}_{2}^{+}+\mathbf{N}_{2}$ & $\rightarrow$ & $\mathrm{NO}^{+}+\mathrm{NO}$ \\
\hline R12 & $\mathrm{O}_{2}^{+}+\mathrm{NO}$ & $\rightarrow$ & $\mathrm{NO}^{+}+\mathrm{O}_{2}$ \\
\hline R13 & $\mathbf{O}_{2}^{+}+\mathbf{N}$ & $\rightarrow$ & $\mathrm{NO}^{+}+\mathrm{O}$ \\
\hline R14 & $\mathbf{O}^{+}+\mathrm{N}_{2}$ & $\rightarrow$ & $\mathrm{NO}^{+}+\mathrm{N}$ \\
\hline R15 & $\mathbf{O}^{+}+\mathbf{O}_{2}$ & $\rightarrow$ & $\mathbf{O}_{2}^{+}+\mathbf{O}$ \\
\hline R16 & $\mathrm{O}^{+}+\mathrm{NO}$ & $\rightarrow$ & $\mathrm{NO}^{+}+\mathrm{O}$ \\
\hline
\end{tabular}

\subsection{Continuity Equation}

The continuity equation for each species $s$ is

$$
\frac{\partial n_{s}}{\partial t}+\nabla \cdot\left(n_{s} u_{s}\right)=P_{s}-L_{s} n_{s}
$$

where $n_{s}$ is the number density of species $s, u_{s}$ is the drift velocity, $P_{s}$ is the ionization production rate, and $L_{s}$ is the ionization loss frequency. This equation is greatly simplified if the divergence in the flow of species $s$ is unimportant and a steady state solution to (1) is sufficient. In this instance, the continuity equation becomes

$$
P_{s}=L_{s} n_{s}
$$

Since, locally, the production rate is equal to the loss rate, equation (2) assumes that the ionosphere is in photochemical equilibrium. The range of validity for (2) in the ionosphere is examined later.

Photodissociation of the neutral atmosphere is the dominant source of ions in the Earth's ionosphere. In the daytime ionosphere, the major source for photodissociation is extreme ultraviolet (EUV) radiation from the Sun. At nighttime, EUV radiation from starlight, resonantly scattered solar radiation, and radiative recombination become important [Strobel et al., 1980]. Another important ionization source is auroral precipitation. As energetic electrons precipitate into the atmosphere, they lose energy via inelastic collisions with the neutral atmosphere and this energy can produce electron-ion pairs. These production sources are included in our model.

The four ions which are most important in the $E$ and lower $F$ regions are $\mathrm{N}_{2}^{+}, \mathrm{O}_{2}^{+}, \mathrm{O}^{+}$, and $\mathrm{NO}^{+}$. The major photochemical reactions for these ions are shown in Table 1 and the rate coefficients for the reactions were obtained from Schunk and Raitt [1980]. The nonlinear nature of these reactions makes a numerical solution necessary. For more details about the numerical model, see the appendix.

\subsection{Momentum Equation}

The momentum equation describes the transfer of momentum from collisions between ions and constituents of the neutral atmosphere. It is these collisions which lead to ionospheric conductivity. A relatively general form for the momentum equation is given by Schunk and Nagy [1980],

$$
\begin{aligned}
n_{s} m_{s} \frac{D_{s} \mathbf{u}_{s}}{D t}+ & \nabla p_{s}+\nabla \cdot \tau_{s}-n_{s} m_{s} \mathbf{G}-n_{s} e_{s}\left[\mathbf{E}+\frac{1}{c} \mathbf{u}_{s} \times \mathbf{B}\right] \\
= & -\sum_{t} n_{s} m_{s} \nu_{s t}\left(\mathbf{u}_{s}-\mathbf{u}_{t}\right) \\
& +\sum_{t} \nu_{s t} \frac{z_{s t} \mu_{s t}}{k T_{s t}}\left[\mathbf{q}_{s}-\frac{\rho_{s}}{\rho_{t}} \mathbf{q}_{t}\right]
\end{aligned}
$$

where $D_{s} / D t=\partial / \partial t+\mathbf{u}_{s} \cdot \nabla$ is the convective derivative of species $s, p_{s}=n_{s} k T_{s}$ is the partial pressure, $m_{s}$ is the mass, $e_{s}$ is the charge, $T_{s}$ is the temperature, $\tau_{s}$ is the stress tensor, $G$ is the acceleration due to gravity, $\mathbf{E}$ is the electric field, $B$ is the magnetic field, $c$ is the speed of light, and $k$ is Boltzmann's constant. In the collision term, which is on the right-hand side of (3), $\rho_{s}=n_{s} m_{s}$ is the mass density, $\mu_{s t}=m_{s} m_{t} /\left(m_{s}+m_{t}\right)$ is the reduced mass, $T_{s t}=\left(m_{t} T_{s}+m_{s} T_{t}\right) /\left(m_{s}+m_{t}\right)$ is the reduced temperature, $q_{s}$ is the heat flow vector, and $\nu_{s t}$ is the momentum transfer collision frequency for gases $s$ and $t$. The quantity $z_{s t}$ is a pure number that is different for different combinations of species $s$ and $t$; representative values are given by $S$ chunk [1977].

In order to obtain an equation for the components of the conductivity tensor,

$$
\sigma=\left|\begin{array}{ccc}
\sigma_{P} & -\sigma_{H} & 0 \\
\sigma_{H} & \sigma_{P} & 0 \\
0 & 0 & \sigma_{0}
\end{array}\right|
$$

where $\sigma_{P}$ is the Pedersen, $\sigma_{H}$ is the Hall and $\sigma_{0}$ is the longitudinal component, it is assumed that the convective derivative, the partial pressure, the stress tensor, and the acceleration due to gravity are insignificant. Additionally, if the heat flow vector on the right-hand side of (3) can be safely ignored, one obtains

$$
\mathbf{E}+\frac{1}{c} \mathbf{u}_{s} \times \mathbf{B}=\sum_{i} \frac{m_{s} \nu_{s t}}{e_{s}}\left(\mathbf{u}_{s}-\mathbf{u}_{t}\right)
$$

Finally, one can obtain the Pedersen and Hall components of the conductivity tensor in the rest frame of the neutral gas (the frame where $\mathbf{u}_{t}=0$ ) from (5) and Ohm's law, $J=\sigma \cdot E$, where $J=$ $\Sigma_{i} e_{i} n_{i} u_{i}-e n_{e} u_{e}$ is the current. These components are

$$
\begin{gathered}
\sigma_{P}=\frac{e n_{e} c}{B}\left(\sum_{i} \frac{C_{i} \nu_{i} / \Omega_{i}}{A_{i}}+\frac{\nu_{e} / \Omega_{e}}{A_{e}}\right) \\
\sigma_{H}=\frac{e n_{e} c}{B}\left(\sum_{i} \frac{C_{i}}{A_{i}}-\frac{1}{A_{e}}\right)
\end{gathered}
$$

where $\nu_{s}=\Sigma_{1} \nu_{s t}$ is the effective collision frequency, $\Omega_{s}=e B / m_{s} c$ is the cyclotron frequency, $C_{i}=e_{i} n_{i} / e n_{e}$, and $A_{s}=1+\nu_{s}^{2} / \Omega_{s}^{2}$.

Once a solution for ionospheric densities has been obtained from (2), one can easily solve for the Pedersen and Hall conductivity components from (6). Examples of solutions to both (2) and (6) are presented in the next two sections for a range of geophysical conditions.

\section{Electron Density}

In this section we compare the results of the photochemical equilibrium model with observations of the Arecibo and Chatanika radars. The choice of these two radars and the times of the observations allow a comparison with data for a wide range of geophysical conditions. The Arecibo observations were made on August 10,1974, during which time the solar cycle was near solar minimum, and the Chatanika observations were made on June 27,1981 , when the solar cycle was near solar 


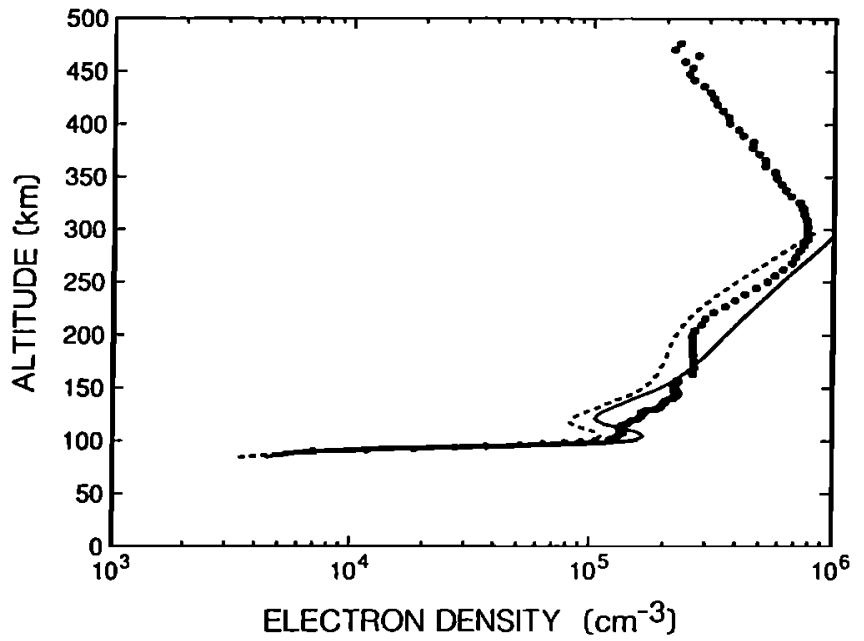

Fig. 1. A comparison of the photochemical equilibrium model with Arecibo observations for an overhead Sun $\left(x=3^{\circ}\right)$. The dots represent radar measurements of electron density, while the solid line represents model results for $F_{10.7}=210$ and the dashed line for $F_{10.7}=90 \times 10^{-22} \mathrm{~W}$ $\mathrm{m}^{-2} \mathrm{~Hz}^{-1}$

maximum. The location of the Arecibo radar allowed a model/ data comparison over a wide range of solar zenith angles, while the location of the Chatanika radar allowed a comparison in the auroral zone. Further information about data acquisition and the mode of operation of the Arecibo radar is given by Emery et al. [1981], while operation of the Chatanika radar during the June 1981 period is covered in detail by Rasmussen et al. [1986].

In Figure 1, a comparison of the photochemical equilibrium model with Arecibo observations is shown. The radar data are plotted as dots and the model results as lines (solid for $\boldsymbol{F}_{10.7}=$ 210 and dashed for $F_{10.7}=90$ ). The two values of $F_{10.7}$ were chosen to show the variation of electron density over a wide range of solar conditions, with the dashed line most closely representing solar activity at the time of the measurements $\left(F_{10.7}\right.$ $=88.3)$. The Sun was nearly directly overhead $\left(\chi=3^{\circ}\right)$ at the time of these measurements (1641:25 UT).

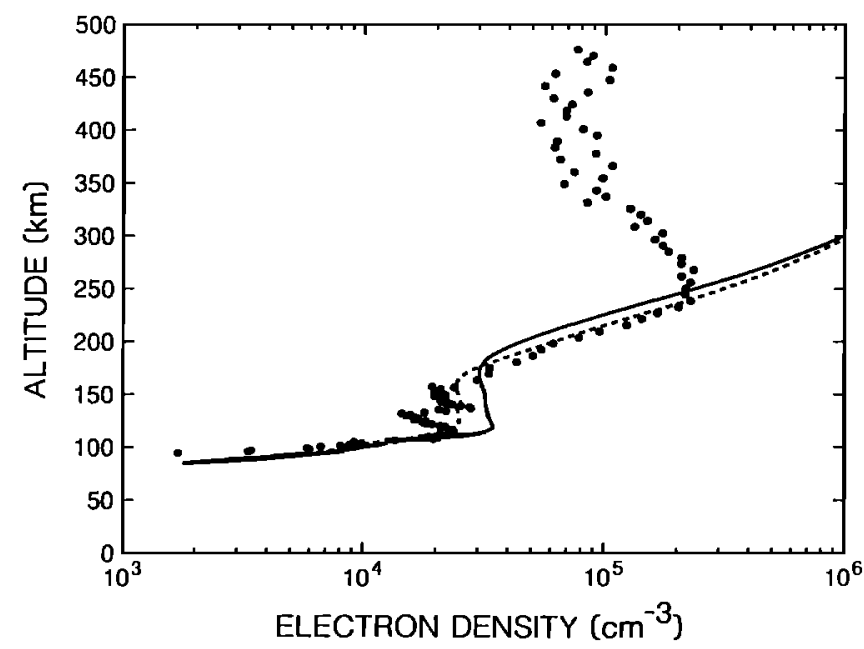

Fig. 2. A comparison results of the photochemical equilibrium model with Arecibo observations for twilight conditions $\left(x=86^{\circ}\right)$. The dots represent radar measurements of electron density, while the solid line represents model results for $F_{10.7}=\mathbf{2 1 0}$ and the dashed line for $F_{10.7}=\mathbf{9 0}$ $\times 10^{-22} \mathrm{~W} \mathrm{~m}^{-2} \mathrm{~Hz}^{-1}$.

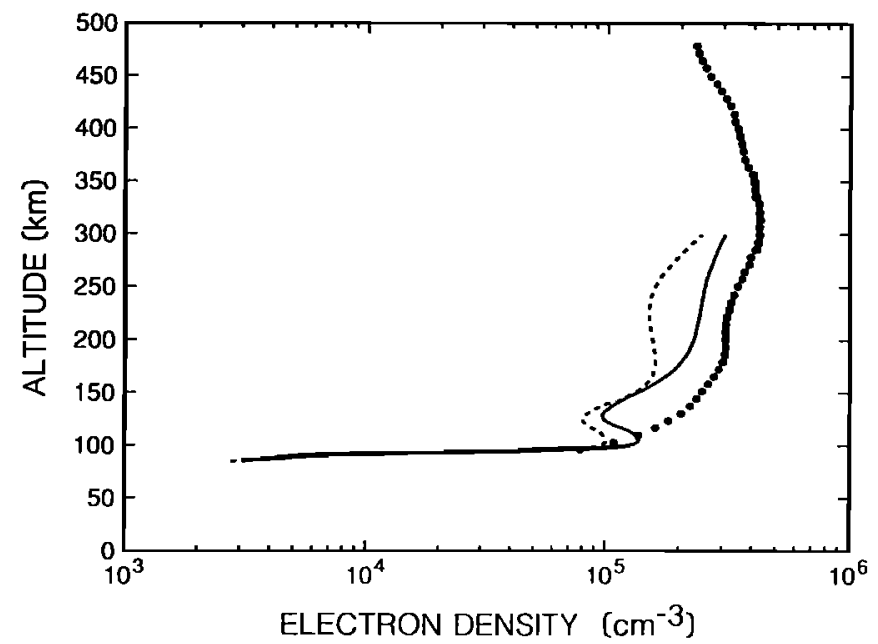

Fig. 3. A comparison of the photochemical equilibrium model with Chatanika observations for a zenith angle of $44^{\circ}$. The dots represent radar measurements of electron density, while the solid line represents model results for $F_{10.7}=210$ and the dashed line for $F_{10.7}=90 \times 10^{-22} \mathrm{~W}$ $\mathrm{m}^{-2} \mathbf{H z}^{-1}$

The dashed line in Figure 1 is seen to very closely match the observations up to an altitude of about $100 \mathrm{~km}$. At this altitude the model results show a peak in electron density due to Lyman $\beta$ ionization of $\mathrm{O}_{2}$. Just above $100 \mathrm{~km}$, the model results fall off sharply with increasing altitude while the observations do not. Although the data show some evidence of a relative maximum at this altitude (seen more clearly in measurements at other times), the model consistently predicts a much stronger fall-off than was measured. From 110 to $200 \mathrm{~km}$, the model underestimates the data by $30-40 \%$.

The model performed much better in the early morning of the same day, as can be seen in Figure $2\left(\chi=86^{\circ}, 1027: 16 \mathrm{UT}\right)$. In this figure the model is again compared with Arecibo measurements (represented by dots), where the dashed line represents the model results $\left(F_{10.7}=90\right)$ for solar conditions near those at the time of the measurements. In this comparison, it is seen that the model predicts remarkably well the ionosphere up to 250 $\mathrm{km}$. However, just $20 \mathrm{~min}$ later, the model again underestimates (not shown) the electron density in a manner similar to that shown in Figure 1. It should be pointed out that above $200 \mathrm{~km}$, diffusion becomes important and, in general, the assumption of photochemical equilibrium is not valid much above $200-220$ $\mathrm{km}$. This is especially evident above $250 \mathrm{~km}$ in Figure 2, where the model results depart significantly from the measurements. Thus, in the calculations of conductivity which follow in the next section, the photochemical equilibrium model was not used above $220 \mathrm{~km}$.

A comparison of the model with Chatanika measurements is shown in Figure 3. The time during which these measurements were taken was near local noon $\left(\chi=44^{\circ}, 2308: 22\right.$ UT) on June 27,1981 , when the solar cycle was near maximum $\left(F_{10.7}=193\right)$. Therefore, contrary to the previous two figures, the solid line now most nearly represents solar conditions at the time of the measurements. Similar to the results shown in Figure 1, the model predicts a well-defined peak at $100 \mathrm{~km}$ and the predictions compare favorably with the measurements up to this altitude. However, above this altitude, the model underestimates the densities by $40-50 \%$.

As was seen in the three previous figures, the model under- 


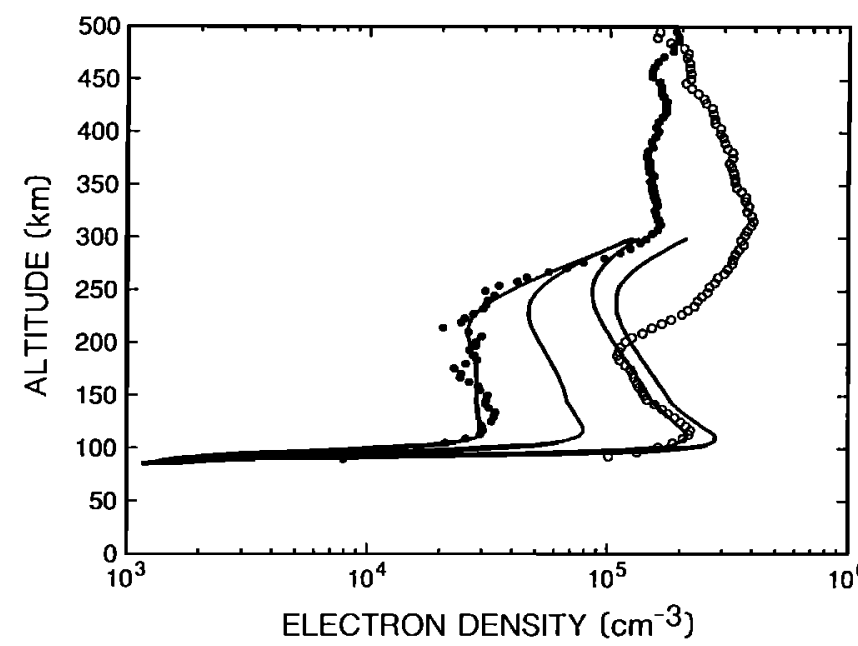

Fig. 4. A comparison of the photochemical equilibrium model with Chatanika observations during auroral activity. The dots represent radar measurements of electron density at 1101:22 UT and $63^{\circ}$ dipole latitude, while the circles represent radar measurements taken about 4 min later and $4^{\circ}$ further north. The four curves show model results for different levels of auroral activity.

estimates electron densities produced by solar illumination at altitudes from 110 to $200 \mathrm{~km}$. This has been a problem with previous ionospheric models as well. For example, Heroux et al. [1974] underestimated electron densities by $30 \%$ in this region and Torr et al. [1979] underestimated $F_{1}$ densities by as much as $50 \%$. However, ionospheric modeling depends on a knowledge of several parameters, many of which are not precisely known. Torr et al. [1979] performed a sensitivity analysis and found that it is probably not possible to model the average behavior of the ionosphere to an accuracy of better than $\pm 60 \%$ owing to uncertainties in solar EUV fluxes, chemical reaction rates, collision cross sections, etc. Of these, uncertainties in the solar EUV flux are probably the most critical. Most of the measurements of the solar EUV flux are from satellites and it is difficult to establish the absolute calibration of an instrument on a satellite because the sensitivity of the instrument changes during long exposure to the space environment [Lean and Skumanich, 1983]. For instance, Hinteregger et al. [1981] have revised earlier solar EUV measurements by as much as $60 \%$. These changes were found to lead to a decrease in ionization frequencies by $12-33 \%$ [Torr and Torr, 1985]. Thus, although our model tends to consistently underestimate the electron density, further improvement is probably not possible at this time.

The final electron density comparison is shown in Figure 4. Here, the ionosphere below $200 \mathrm{~km}$ is maintained by auroral precipitation rather than by solar illumination. The dots represent Chatanika measurements at a dipole latitude of $63^{\circ}$ and a magnetic local time of 23.3 hours (1101:22 UT). The four solid curves represent model results for different levels of auroral activity. The model requires as an input both the flux and the characteristic energy of the electron precipitation. These inputs were provided by the Spiro et al. [1982] empirical model, which sorts precipitation flux and energy according to the $A E$ index. The four curves represent $A E$ values for each of the four bins of the Spiro model, the lowest (leftmost) curve corresponds to the lowest $A E$ bin and each of the remaining curves (moving from left to right) corresponds to progressively higher $A E$ values.

As can be seen in Figure 4, the lowest density curve repro- duces the measurements (solid dots) remarkably well. However, the $A E$ index at the time of the measurements was 174 , so the second curve with a peak $E$ region density of $8 \times 10^{4} \mathrm{~cm}^{-3}$, most nearly represents conditions at the time of the measurements. The predicted densities are roughly a factor of 3 times higher than the measurements. However, the binned values of Spiro et al. [1982] represent average conditions only, and it is not known what the actual flux and energy for this particular auroral event were. It should be noted that a particular event may vary from the average by a large margin. This can be seen by examining the circles in Figure 4, which represent measurements made by the Chatanika radar just $4 \mathrm{~min}$ later and $4^{\circ}$ further north than the measurements represented by the dots. In this instance, the measurements are very closely modeled by the third $A E$ bin (300<AE<600). Note that, although the circles represent measurements at a latitude different from that used to obtain the third curve, in this case the latitude difference has a negligible effect on the model results.

Because measurements of electron flux and energy were not available for the two data sets plotted in Figure 4, we cannot make definitive conclusions about the absolute density scale of the model results. However, the model seems to accurately predict the shape of the electron density curves, and indications are that the scaling is probably correct as well. In an earlier study, Vondrak and Robinson [1985] had access to AE-C measurements of precipitation fluxes and energies in regions of the ionosphere where Chatanika was making simultaneous measurements. They found, also using the Rees [1963] method to obtain auroral production rates, that calculated densities were within $25 \%$ of the measured densities.

\section{Conductivity}

Having compared the photochemical equilibrium model with $E$ and lower $F$ region densities, we now examine values of height-integrated conductivity, which are calculated from the ion densities predicted by the model. The variations of conductance with solar zenith angle, solar flux, and auroral activity are examined. Also, the importance of the $F$ region ionosphere to conductance is considered.

The dependence of conductance on the solar zenith angle is shown in Figure 5, where model conductances are compared with Arecibo and Chatanika measurements (inferred from density data). The dots (circles) represent measurements by the Arecibo radar of height-integrated Hall (Pedersen) conductivity. The solid and open rectangles represent Chatanika measurements made on June 16, 1977, of Hall and Pedersen conductances, respectively. The measurements of the two radars can be compared because they were taken during periods of similar solar activity and the Chatanika measurements have been scaled to take into account the magnetic field strength at Arecibo. The two solid lines represent the results of the model. For a relatively high Sun, it is seen that the Hall conductance is underestimated by the model by $20-25 \%$, while the Pedersen conductance is underestimated by $30-35 \%$. This underestimation of conductance is due to the underestimation of electron density referred to earlier. Note, however, that many of the measurements of Hall conductance at Arecibo for $\chi>45^{\circ}$ are close to the predicted values. These measurements were taken in the afternoon and are roughly $20 \%$ less than the measurements made in the morning.

The two dashed curves represent the empirical model results of Vickrey et al. [1981] and Mehta [1978]. The bottom dashed 


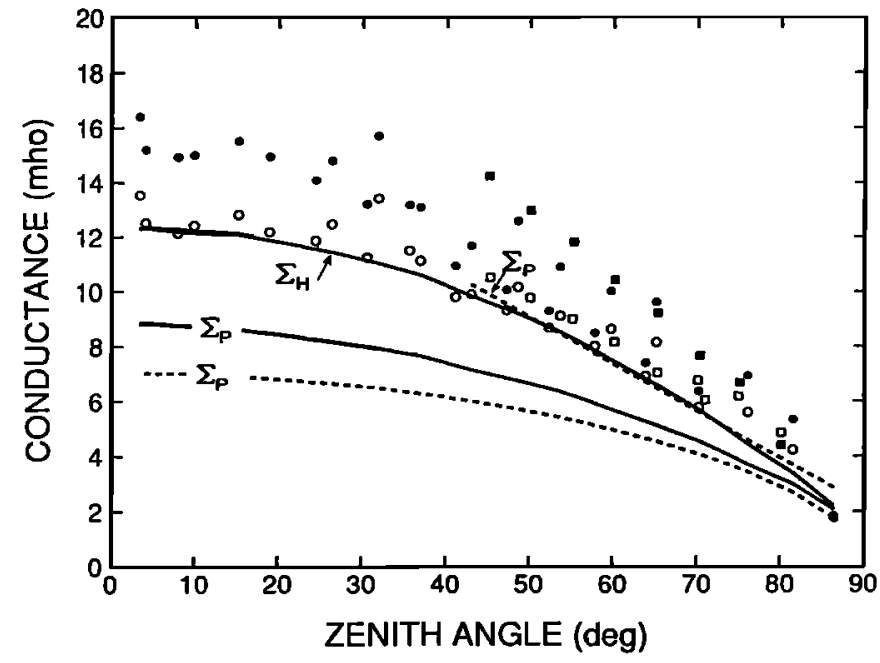

Fig. 5. Conductance versus solar zenith angle for both the photochemical equilibrium model and Arecibo and Chatanika measurements. The dots represent Arecibo measurements of Hall conductance and the circles Pedersen conductance. The solid and open rectangles represent Chatanika measurements of Hall and Pedersen conductances, respectively. The solid lines show our model predictions, and the dashed curves are empirical models results.

curve in Figure 5 is a plot of the function $5[\cos (\chi)]^{1 / 2}$ (multiplied by a constant factor to account for the difference in magnetic field strength between Chatanika and Arecibo). This is the function found by Vickrey et al. [1981] to fit measurements of Pedersen conductance made by the Chatanika radar. The shape of this function roughly fits the trend of the data, but the magnitude is off. The measurements of Pedersen conductance made by Vickrey et al. [1981] differ from the Arecibo measurements by $40-45 \%$. It is unclear why the Vickrey et al. [1981] measurements of Pedersen conductance are so much lower than the measurements shown in Figure 5. It might be helpful to point out that the Vickrey et al. [1981] results represent only one day of data (April 6, 1977) and their empirical results represent a fit to the lowest conductivity values obtained during this day. Also, the model neutral atmosphere used in that study was probably only accurate to within a factor of 2 .

The straight line segment (top dashed curve) is a plot of the function, $12.579-0.122 \chi$ (also multiplied by a constant factor to account for differences in magnetic field strength), which was found by Mehta [1978] to fit measurements of Pedersen conductance made with the Chatanika radar for solar zenith angles in the range $45^{\circ} \leq \chi \leq 95^{\circ}$. This empirical model fits the Arecibo measurements very well. However, this is fortuitous in that Mehta [1978] used collision frequencies that were a factor of 2 too high [Vickrey et al., 1981]. When the collision frequencies are corrected, the results of Mehta [1978] are close to those of Vickrey et al. [1981].

An important point to consider when comparing measurements of conductance is that the thermospheric model used can appreciably affect the results. For instance, we have tested the sensitivity of the thermospheric model on conductance, by varying the $F_{10.7}$ input to the MSIS-83 model. We used the electron density profile plotted in Figure 1 and found a 25\% variation in conductance as the thermosphere densities changed in response to solar cycle variations (the electron density remained fixed). Most of the previous studies of conductance used the $1000 \mathrm{~K}$ thermosphere of Banks and Kockarts [1973] [e.g., Brekke et al.,
1974; Horwitz et al., 1978; Vickrey et al., 1981], while the MSIS-83 model Hedin [1983] was used in this study.

The ratio of the height-integrated Hall to Pedersen conductivities $\left(\Sigma_{H} / \Sigma_{P}\right)$ is important. Earlier studies have found this ratio to be about 2 in solar-illuminated regions outside of auroral precipitation [Brekke et al., 1974; Mehta, 1978; Vickrey et al., 1981]. However, we found $\Sigma_{H} / \Sigma_{P}$ to be of the order of 1.1-1.3 for both the model results and the Arecibo measurements (also for the Chatanika measurements in regions free from auroral precipitation). This decrease in the $\Sigma_{H} / \Sigma_{P}$ ratio is primarily due to our increased values of Pedersen conductance, as earlier measurements of Hall conductance are similar to our results.

Next, the dependence of conductance on solar cycle is considered. The output of the Sun varies with solar cycle, especially at wavelengths short enough to ionize the neutral atmosphere. Therefore, one would expect the conductivity of the ionosphere to change during a solar cycle. This is indeed the case, as can be seen in Figure 6, where the conductance from our model is plotted as a function of the solar $10.7 \mathrm{~cm}$ flux. The Hall and Pedersen conductances (solid and dashed lines, respectively) increase by about a factor of 1.6 from solar minimum to solar maximum. Also shown in Figure 6 are Chatanika measurements of Hall (dots) and Pedersen (circles) conductances taken on 13 separate days during the period August 11, 1976, through July 14, 1981 (see Table 2). Information about data acquisition and analysis for these days can be obtained from Johnson et al. [1987]. These measurements represent the solar contribution to conductance at a solar zenith angle of $60^{\circ}$ and are seen to increase in roughly the same manner with the $10.7 \mathrm{~cm}$ flux as does the modeled conductances. However, again as in Figure 5, the model is seen to underestimate the measured conductances.

Auroral precipitation is also a significant source of ionization and the effect of the characteristic energy of the precipitation on conductance is shown in Figure 7. The solid curves are from our chemical-equilibrium model, while the dashed curves are from Vickrey et al. [1981] and are shown for comparison. For all curves the energy flux was fixed at $1 \mathrm{erg} / \mathrm{cm}^{2} / \mathrm{s}$. At lower

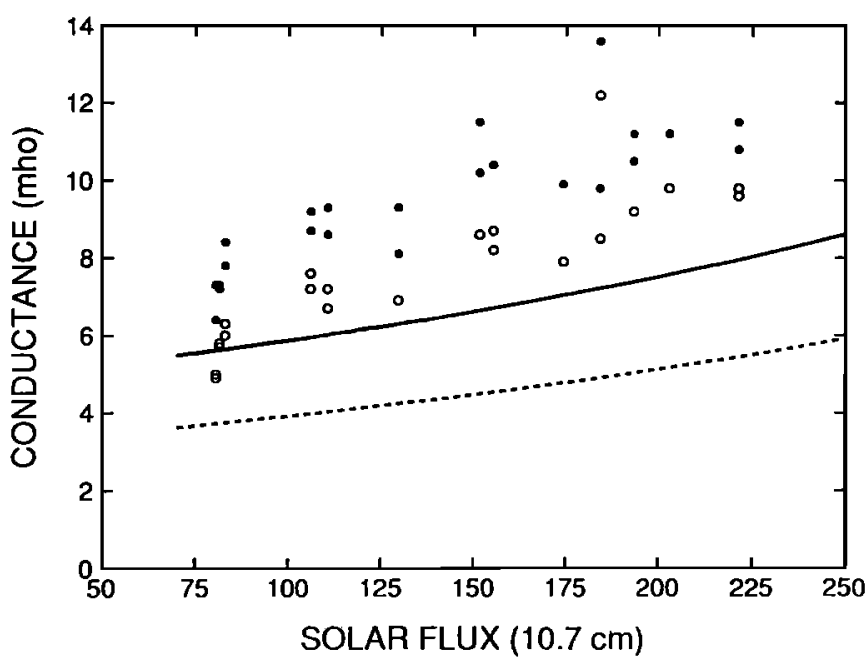

Fig. 6. Conductance versus the solar $10.7-\mathrm{cm}$ flux. The dots and the circles represent Chatanika measurements of Hall and Pedersen conductances, respectively. The solid line represents model predictions of Hall conductance, and the dashed line Pedersen conductance. The solar flux has units of $10^{-22} \mathrm{~W} \mathrm{~m}^{-2} \mathrm{~Hz}^{-1}$. 
TABLE 2. Chatanika Measurements

\begin{tabular}{lrc}
\hline \multicolumn{1}{c}{ Day } & Ap & Solar Flux, $S_{\boldsymbol{a}}$ \\
\hline August 11, 1976 & 5 & 80.4 \\
May 18, 1977 & 11 & 82.9 \\
June 16, 1977 & 10 & 81.4 \\
May 10, 1978 & 10 & 129.5 \\
June 7, 1978 & 11 & 110.6 \\
July 12, 1978 & 4 & 174.2 \\
August 1, 1978 & 4 & 106.0 \\
June 20,1979 & 8 & 151.5 \\
May 14, 1980 & 17 & 202.9 \\
July 9, 1980 & 8 & 155.2 \\
August 13, 1980 & 6 & 193.3 \\
May 13, 1981 & 10 & 221.3 \\
July 14, 1981 & 7 & 184.2 \\
\hline
\end{tabular}

energies, the precipitation produces ionization at higher altitudes, and therefore the Pedersen conductance is larger than the Hall conductance. As the energy of the electron precipitation increases, it reaches to lower altitudes in the atmosphere. This acts to produce a peak in the Pedersen conductance just under 2 $\mathrm{keV}$ and a peak in the Hall conductance at about $6 \mathrm{keV}$. For ionization caused by auroral precipitation, the results of our model are similar to, although somewhat lower than, the results of Vickrey et al. [1981].

Having examined the dependence of conductance on the solar zenith angle, the solar $10.7 \mathrm{~cm}$-flux, and the characteristic energy of auroral precipitation, we now show plots of ionospheric conductance along the dawn-dusk meridian. In Figure 8 the Hall (solid line) and Pedersen (dashed line) conductances are plotted as a function of colatitude for quiet auroral activity $(A E=80)$ and for solar minimum $\left(F_{10.7}=70\right)$. The day chosen was solar equinox in the fall and the universal time was chosen such that the terminator coincided with the geomagnetic pole. The dayside portion of the graph is represented by negative colatitude. As can be seen in the figure, both the Hall and Pedersen conductances rise rapidly to a peak at the dayside equator, where the Sun is directly overhead. Part of the rise is

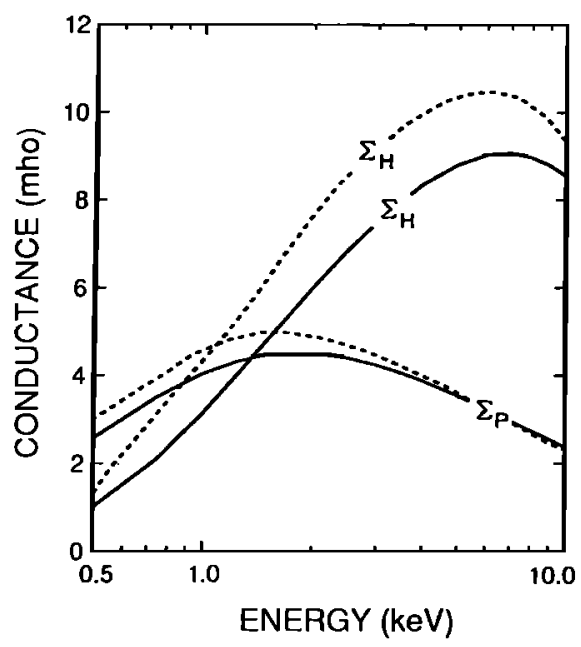

Fig. 7. Conductance versus the energy of precipitating electrons. The dashed lines are from Vickrey et al. [1981], while the solid lines show the photochemical equilibrium model results.

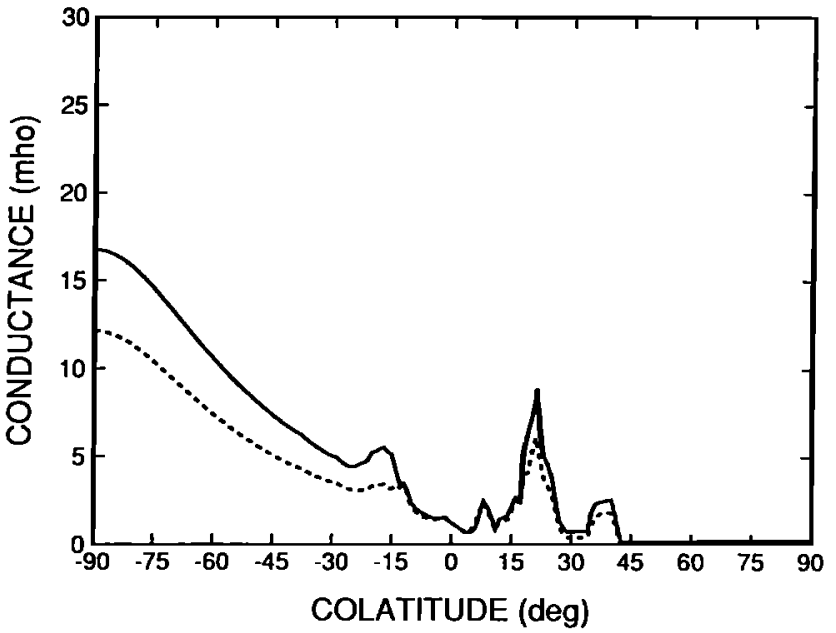

Fig. 8. Conductance along the dawn-dusk meridian for solar-cycle minimum and quiet auroral conditions. The solid line represents model predictions of Hall conductance, and the dashed line Pedersen conductance. The dayside part of the plot corresponds to negative colatitudes.

due to a decreasing solar zenith angle and part of the rise is attributed to a decrease in the magnetic field strength (dipole model) at lower latitudes.

On the nightside the conductance decays to about 0.1 mhos for the Pedersen conductance and about $\mathbf{0 . 2}$ mhos for the Hall conductance. These levels of nighttime conductance are the same as those estimated by Wallis and Budzinski [1981], although measurements can be 2-3 times higher and can vary considerably from the mean [Harper and Walker, 1977; Rowe and Mathews, 1973]. The maintenance processes for nighttime conductivity are principally starlight and resonantly scattered solar radiation.

The effect of auroral activity is shown in Figure 8, where an increase in conductance is seen around $20^{\circ}$ colatitude on both the dayside and nightside regions. The jagged nature of the conductance in the auroral zone is due to the Spiro et al. [1982] empirical model of precipitation flux and characteristic energy used in the chemical-equilibrium model. For quiet auroral activity, the Hall conductance reaches a maximum of about 10 mhos. For active auroral conditions, the conductance increases dramatically, as can be seen in Figure 9, where an $F_{10.7}$ of 210 and an $A E$ of 620 were chosen to reflect solar maximum and strong geomagnetic activity, respectively (the day and universal time are the same as in Figure 8). The Pedersen conductance increases by a factor of about 2.5 , and the Hall conductance increases even more due to the increase in the energy of the precipitation.

One of the most striking features of Figure 9 is the high level of conductance at low latitudes due to the relatively high solar fluxes during solar maximum. The Pedersen conductance is about 20 mhos and the Hall conductance is almost 26 mhos. This is $2-4$ times higher than conductances measured at highlatitude sites during solar minimum conditions [Mehta, 1978; Vickrey et al., 1981]. Also, keep in mind that the photochemical equilibrium model slightly underestimates conductance, as discussed earlier, so ionospheric conductance is expected to be even larger than shown in the plot.

Next, we consider one of the limitations of the model. Since chemical equilibrium is assumed, vertical diffusion of ionization is not included, and thus, the model results are only valid up to 


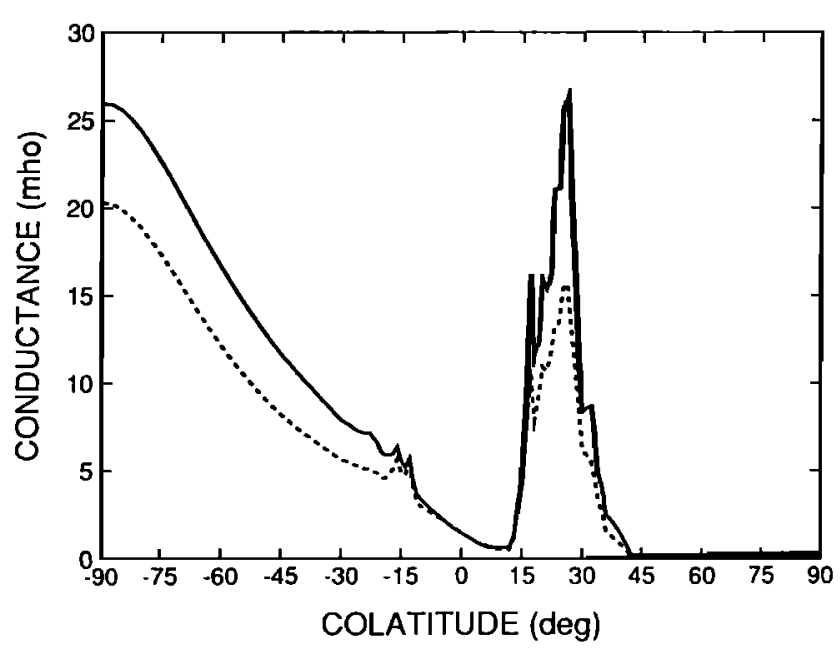

Fig. 9. Conductance along the dawn-dusk meridian for solar-cycle maximum and active auroral conditions. The solid line represents model predictions of Hall conductance, and the dashed line Pedersen conductance. The dayside part of the plot corresponds to negative colatitudes.

approximately $200-220 \mathrm{~km}$ during the daytime. We have not exceeded this altitude in the preceding calculations. To see the contribution of the upper ionosphere, we ran our $F$ region model, which includes the effects of diffusion [Schunk and Walker, 1973; Schunk and Raitt, 1980], for a diurnal cycle at midlatitudes ( $45^{\circ}$ dipole latitude). The solar fluxes used for this run were for solar maximum conditions $\left(F_{10.7}=210\right)$ and an $A E$ of 620 was used. The chemical equilibrium model was also run for the same conditions and the results of the two models were joined to find the total conductance from $85 \mathrm{~km}$ to $400 \mathrm{~km}$. The relative contributions of ionospheric densities above 170 $\mathrm{km}$ (solid line) and above $220 \mathrm{~km}$ (dashed line) to the Pedersen conductance are shown in Figure 10. During the daytime, nearly $40 \%$ of the contribution to the height-integrated Pedersen conductivity is above $170 \mathrm{~km}$, and roughly $25 \%$ of the contribution is due to the ionosphere above $220 \mathrm{~km}$. At dusk (near 1900 MLT) the high-altitude contribution increases dramatically because of the longer time constants involved at higher altitudes. The significant contribution of the $F$ region at night to Pedersen conductance has been substantiated by measurements at Arecibo [ Harper and Walker 1977].

For solar minimum conditions, the relative contribution of the high-altitude portion of the ionosphere is less. During daylight hours, the region of the ionosphere above $170 \mathrm{~km}$ contributes roughly $20 \%$ to the total height-integrated Pedersen conductivity, increasing to $60 \%$ at dusk. The contribution above $220 \mathrm{~km}$ is only $5 \%$ during daylight hours.

Finally, we offer simple formulas for the solar contribution to height-integrated conductivity. The "functional dependence" of conductance on the solar zenith angle and on the solar $10.7-\mathrm{cm}$ flux was obtained by fitting second-order polynomials to our model results. These functions were then scaled to fit the measurements of the Arecibo and Chatanika radars. The formulas are

$$
\begin{aligned}
& \Sigma_{P}=\frac{4.5}{B}\left(1-0.85 v^{2}\right)\left(1+0.15 u+0.05 u^{2}\right) \\
& \Sigma_{H}=\frac{5.6}{B}\left(1-0.9 v^{2}\right)\left(1+0.15 u+0.05 u^{2}\right)
\end{aligned}
$$

where $v=\chi / 90^{\circ}, u=F_{10.7} / 90$, and where the solar zenith angle is in degrees, the $10.7-\mathrm{cm}$ flux is in units of $10^{-22} \mathrm{~W} \mathrm{~m}^{-2} \mathrm{~Hz}^{-1}$, the magnetic field strength is in gauss, and the conductance is in mhos. These formulas are applicable within the ranges $0 \leq \chi \leq$ $85^{\circ}$ and $70 \leq F_{10.7} \leq 250$. Equations $(7 a)$ and $(7 b)$ were found to fit very nicely the trend in the data and to be within $\pm 20 \%$ in magnitude.

\section{ConClusions}

A photochemical equilibrium model of the high-latitude ionosphere has been developed. This model provides values of electron density from $85 \mathrm{~km}$ to approximately $220 \mathrm{~km}$. A comparison of the model with Arecibo and Chatanika radar observations was made to check the model during periods of solar minimum and solar maximum. Certain observations by the Chatanika radar allowed the model to be compared with measurements in regions of electron precipitation as well.

The model accurately predicts electron densities up to about $110 \mathrm{~km}$ for an illuminated ionosphere. The production source at this altitude is principally due to ionization of $\mathrm{O}_{2}$ by the Lyman $\beta$ line of solar hydrogen. Between 125 and $175 \mathrm{~km}$ the model consistently underestimates the electron content by $40-50 \%$. Previous $E$ region models have also underestimated the electron density in this region of the ionosphere. Because of the uncertainties in the values of many of the inputs to the models (such as the solar EUV flux), it is probably not possible to model the average behavior of the ionosphere to an accuracy better than this [Torr et al., 1979].

The model accurately predicts levels of ionization created by electron precipitation. Both the shape (with respect to altitude) and the range of densities of the measurements were well reproduced, assuming a gaussian energy distribution with the characteristic energy and flux given by the empirical model of Spiro $e t$ al. [1982]. Since no measurements of electron flux and energy were available, it is uncertain if the scaling of the model results is correct in regions of electron precipitation. However, in an earlier study, Vondrak and Robinson [1985] had access to AE-C measurements of precipitation fluxes and energies in regions of the ionosphere where Chatanika was making simultaneous measurements. They found, also using the Rees [1963] method

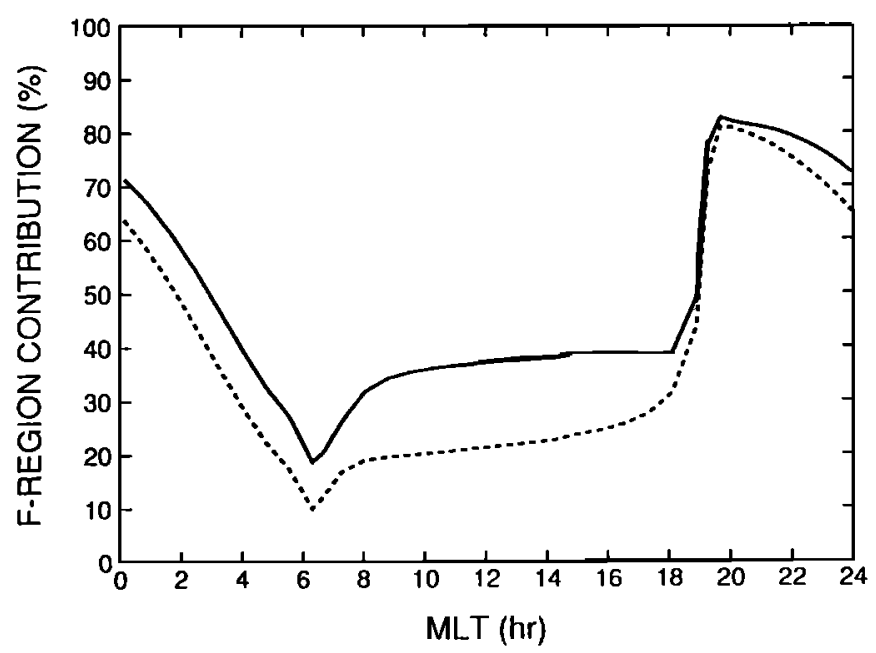

Fig. 10. Contribution of the $F$ region densities to the total heightintegrated Pedersen conductivity. The solid line is the relative contribution above $170 \mathrm{~km}$, and the dashed line is the relative contribution above $220 \mathrm{~km}$. 
to obtain auroral production rates, that calculated densities were within $25 \%$ of the measured densities.

The ability to predict densities in the $E$ and lower $F$ regions allowed a calculation of ionospheric conductivity to be made. A comparison of model results with radar observations showed that the model underestimates the Pedersen conductance by approximately $30-40 \%$ and the Hall conductance by approximately $20-30 \%$ in sunlit portions of the ionosphere. Even with this underestimation, very large conductances were calculated during solar maximum conditions at low latitudes $\left(\Sigma_{P} \simeq 20\right.$, $\Sigma_{H} \simeq 26$ mhos). The conductance was found to increase from solar minimum conditions to solar maximum conditions by a factor of 1.6 .

Since this model assumes photochemical equilibrium, it is only valid up to approximately $200-220 \mathrm{~km}$. The relative contribution of the $F$ region to height-integrated conductivity was calculated using predictions from our $F$ region model. The contribution of the $\boldsymbol{F}$ region to the height-integrated Hall conductivity is insignificant, but the contribution (above $220 \mathrm{~km}$ ) to the height-integrated Pedersen conductivity is $20-25 \%$ during the daytime, increasing to $80 \%$ at dusk. These calculations were done for solar maximum conditions; for solar minimum conditions the relative contribution of the $F$ region is less. This may explain part of the difference in the ratio of height-integrated Hall to Pedersen conductivity obtained from our model and those found in previous studies (other possibilities include differences in modeled collision frequencies and the use of different model atmospheres). Many of the previous studies of conductivity only integrated their measurements up to $170 \mathrm{~km}$. We found that nearly $40 \%$ of the contribution to Pedersen conductance is from the ionosphere above $170 \mathrm{~km}$ for solar maximum (20\% for solar minimum). Thus, we found a Hall to Pedersen ratio of 1.1-1.3, while previous studies have found a ratio of 2.0 [Brekke et al., 1974; Mehta, 1978; Vickrey et al., 1981]. On the other hand, in regions of electron precipitation, our results are within 10-15\% of the calculations of Vickrey et al. [1981].

\section{APPENDIX}

In this appendix, a brief description of each of the major subroutines of the model is given. This is principally done in order to provide a description of the various inputs (such as the model for electron temperature) that are required for the photochemical equilibrium solution. Also, since we are planning to distribute the FORTRAN source code for this model, a description of the subroutines will be helpful to anyone who wishes to use the model.

\section{NeutralAtm( )}

This subroutine returns the values of the neutral constituents, $\mathrm{N}_{2}, \mathrm{O}_{2}, \mathrm{O}, \mathrm{NO}$, and $\mathrm{N}$, of the atmosphere plus the neutral temperature as a function of altitude. The atmospheric $\mathrm{N}_{2}, \mathrm{O}_{2}, \mathrm{O}$ densities as well as the neutral temperature are obtained from the MSIS model atmosphere Hedin [1983]. Daytime and nighttime profiles of nitric oxide and atomic nitrogen were estimated from the results of Ogawa and Shimazaki [1975].

\section{Production( )}

Production rate profiles for the ionospheric constituents, $\mathbf{N}_{2}^{+}$, $\mathrm{O}_{2}^{+}, \mathrm{O}^{+}$, and $\mathrm{NO}^{+}$are calculated in this subroutine. The two major subroutines called by production are described below.

\section{PhotoProd( )}

This subroutine calculates production rates due to EUV radi- ation from the Sun, which is the main source for ionization in the ionosphere. The calculation of the photoionization rates requires a knowledge of the number densities of the neutral constituents, $n_{n}$, as a function of altitude $z$, the absorption $\sigma_{n}^{(a)}(\lambda)$ and ionization $\sigma_{n}^{(i)}(\lambda)$ cross sections of these constituents as a function of wavelength $\lambda$, and the spectrum of solar radiation incident on the top of the atmosphere $I_{\infty}(\lambda)$. In terms of these quantities, the photoelectron production rate is given by

$$
P_{e}(E, z)=\sum_{l} \sum_{n} n_{n}(z) \int_{0}^{\infty} d \lambda I_{\infty}(\lambda) \sigma_{n}^{(i)}(\lambda) \exp [-\tau(\lambda, z)]
$$

where the optical depth $\tau$ is given by

$$
\tau(\lambda, z)=\sum_{n} \sigma_{n}^{(a)}(\lambda) n_{n}(z) H_{n} \operatorname{ch}\left(R_{n}, \chi\right)
$$

and where

$$
\begin{gathered}
H_{n}=k T_{n} / m_{n} g \\
R_{n}=\left(R_{e}+z\right) / H_{n}
\end{gathered}
$$

In (A1)-(A4), $R_{e}$ is the radius of the earth, $\chi$ is the solar zenith angle, and $\operatorname{ch}\left(R_{n}, \chi\right)$ is the Chapman grazing incidence function Chapman [1931]. Approximate expressions for the Chapman function, valid for both large and small solar zenith angles, have been presented by Smith and Smith [1972]. For $\chi<80^{\circ}$ the Chapman function can be replaced by sec $\chi$ in every term in the summation in (A2).

The ultraviolet spectrum was divided into 39 wavelength bins. The wavelength bins are composed of the 37 bins used by Torr and Torr [1985], plus a 31-50 $\AA$ bin and the Lyman $\alpha$ line. The solar fluxes for these bins were obtained from Hinteregger et al. [1981] [see Torr and Torr, 1985] with the 31-50 A flux estimated from Banks and Kockarts [1973] and the Lyman $\alpha$ flux from Bossy and Nicolet [1981]. The photoionization and photoabsorption cross sections were obtained from Kirby et al. [1979] [see Torr and Torr, 1985] with the 31-50 $\AA$ cross sections estimated from Banks and Kockarts [1973] and the Lyman $\alpha$ cross sections from Watanabe et al. [1967]. The effect of photoelectrons was roughly estimated by assuming that an ion is created locally for each $35 \mathrm{eV}$ of energy in the shorter wavelength bins. Nighttime production rates were estimated from Strobel et al. [1980] and include starlight, resonantly scattered solar radiation, and recombination radiation.

\section{AuroralProd( )}

Another source of ionization is auroral precipitation and this source is calculated in this subroutine. As energetic electrons precipitate into the atmosphere, they lose energy via inelastic collisions with the neutral atmosphere which can produce electron-ion pairs. The auroral production rate $\boldsymbol{P}_{\boldsymbol{a}}$ is

$$
P_{a}\left(\epsilon, F_{e}\right)=\frac{F_{e}}{r_{0}} \Gamma(d / D) \frac{\epsilon n_{n}(d)}{\Delta \epsilon n_{n}(D)}
$$

where $\epsilon$ is the initial, monoenergetic electron energy $(\mathrm{keV}), F_{e}$ is the auroral electron flux (number $/ \mathrm{cm}^{2} \mathrm{~s}$ ), $\Delta \epsilon[=0.035 \mathrm{keV}]$ is the mean energy loss per ion pair formed, $n_{n}(d)$ and $n_{n}(D)$ are the number densities of ionizable atoms or molecules $\left(\mathrm{cm}^{-3}\right)$, and $\Gamma(d / D)$ is the fractional, energy-dissipation function Rees [1963]. The variable $d$ is the atmospheric depth $\left(\mathrm{g} / \mathrm{cm}^{2}\right)$ at height $z$, while $D\left[=4.57 \times 10^{-6} \epsilon^{1.75}\right]$ is the atmospheric depth at maximum penetration of the energetic electrons. The form of $\Gamma(d / D)$ is from Figure 1 of Rees [1963], assuming an isotropic distribution of pitch angles from $0^{\circ}$ to $80^{\circ}$. The range (atm-cm) is $r_{0}=$ $D / \rho_{n}$ where $\rho_{n}$ is the mass density $\left(\mathrm{g} / \mathrm{cm}^{3}\right)$ at the lowest altitude of penetration. 
This method requires the characteristic energy and flux of the precipitation. These inputs were obtained from the empirical model of Spiro et al. [1982]. The energy distribution of the precipitation was assumed to be Gaussian and 30 monoenergetic bins from $0.25-45.0 \mathrm{keV}$ were used to model the Gaussian distribution.

\section{Temperature( )}

In this subroutine, profiles of the effective temperature [McFarland et al., 1973; Schunk et al., 1975] and the electron temperature are obtained. At all altitudes the effective temperature is set equal to the neutral temperature. Below $140 \mathrm{~km}$, the electron temperature is also set equal to the neutral temperature, while above $140 \mathrm{~km}$, the electron temperature profiles from Rasmussen et al. [1986] are used.

\section{IonDensity( )}

This subroutine calculates altitude profiles of the ion densities, $\mathrm{N}_{2}^{+}, \mathrm{O}_{2}^{+}, \mathrm{O}^{+}$, and $\mathrm{NO}^{+}$, assuming photochemical equilibrium. Because the set of equations describing the reactions are numerically stiff, a solution is obtained by iterating between two sets of reactions: the reactions involving $\mathrm{N}_{2}^{+}, \mathrm{O}_{2}^{+}, \mathrm{O}^{+}$only, and the much slower reactions involving $\mathrm{NO}^{+}$. The rate coefficients were obtained from the tabulation of Schunk and Raitt [1980].

\section{Conductivity( )}

This subroutine calculates altitude profiles of Pedersen and Hall conductivities given neutral and ion densities and the temperature of the neutrals, ions, and electrons. The collision frequencies needed by this subroutine are from Schunk and Nagy [1980].

\section{HeightInt( )}

Finally, the height-integrated conductivities are obtained by a call to this function, which uses the trapezoidal rule to integrate in height Atkinson [1978].

Acknowledgments. This research was supported by AFOSR contract F49620-86-C-0109 and NASA grant NAGW-77 to Utah State University. The SRI portion of the research was supported by NSF grant ATM 85-16436. We also wish to acknowledge the efforts of Robert Harper for his work in collecting the Arecibo data and Barbara Emery for her work in transfering it to the NCAR Database.

The Editor thanks two referees for their assistance in evaluating this paper.

\section{REFERENCES}

Atkinson, K. E., An Introduction to Numerical Analysis, John Wiley, New York, 1978.

Banks, P. M., and G. Kockarts, Aeronomy, Academic, San Diego, Calif., 1973.

Bossy, L., and M. Nicolet, On the variability of Lyman-alpha with solar activity, Planet. Space Sci., 29, 907-914, 1981.

Brekke, A., J. R. Doupnik, and P. M. Banks, Incoherent scatter measurements of $E$ region conductivities and currents in the auroral zone, J. Geophys. Res., 79, 3773-3790, 1974.

Chapman, S., The absorption and dissociative or ionizing effect of monochromatic radiation in an atmosphere on a rotating Earth, II, Grazing incidence, Proc. Phys. Soc., 43, 483-501, 1931.

Emery, B. A., R. M. Harper, S. Ganguly, J. C. G. Walker, and G. A. Giles, Arecibo ionospheric data August 1974-May 1977, NAIC Rep. 163, Nat. Astron. and Ionos. Cent., Ithaca, N. Y., 1981.

Fuller-Rowell, T. J., and D. S. Evans, Height-integrated Pedersen and Hall conductivity patterns inferred from the TIROS-NOAA satellite data, J. Geophys. Res., 92, 7606-7618, 1987.

Harel, M., R. A. Wolf, P. H. Reiff, R. W. Spiro, W. J. Burke, F. J. Rick, and $M$. Smiddy, Quantitative simulation of a magnetospheric substorm, 1, Model logic and overview, J. Geophys. Res., 86, 2217-2241, 1981.
Harper, R. M., and J. C. G. Walker, Comparison of electrical conductivities in the $E$ - and $F$-regions of the nocturnal ionosphere, Planet. Space Sci., 25, 197-199, 1977.

Hedin, A. E., A revised thermospheric model based on mass spectrometer and incoherent scatter data: MSIS-83, J. Geophys. Res., 88, 10,170-10,188, 1983.

Heroux, L., M. Cohen, and J. E. Higgins, Electron densities between 110 and $300 \mathrm{~km}$ derived from solar EUV fluxes of August 23, 1972, J. Geophys. Res., 79, 5237-5244, 1974.

Hinteregger, H. E., K. Fukui, and B. R. Gilson, Observational, reference and model data on solar EUV, from measurements on AE-E, Geophys. Res. Lett., 8, 1147-1150, 1981.

Horwitz, J. L., J. R. Doupnik, and P. M. Banks, Chatanika radar observations of the latitudinal distributions of auroral zone electric fields, conductivities, and currents, J. Geophys. Res., 83, 1463-1481, 1978.

Johnson, R. M., V. B. Wickwar, R. G. Roble, and J. G. Luhmann, NOAA-thermospheric winds at high latitude: Chatanika radar observations, Ann. Geophys., in press, 1987.

Kamide, Y, and S. Matsushita, Simulation studies of ionospheric electric fields and currents in relation to field-aligned currents, 1, Quiet periods, J. Geophys. Res., 84, 4083-4098, 1979.

Kamide, Y., A. D. Richmond, and S. Matsushita, Estimation of ionospheric electric fields, ionospheric currents, and field-aligned currents from ground magnetic records, J. Geophys. Res., 86, 801-813, 1981.

Kamide, Y., J. D. Craven, L. A. Frank, B.-H. Ahn, and S.-I. Akasofu, Modeling substorm current systems using conductivity distributions inferred from DE auroral images, J. Geophys. Res., 91, 11,235$11,256,1986$.

Kirby, K., E. R. Constantinides, S. Babeu, M. Oppenheimer, and G. A. Victor, Photoionization and photoabsorption cross sections of $\mathrm{He}$ $\mathrm{O}, \mathrm{N}_{2}$, and $\mathrm{O}_{2}$ for aeronomic calculations, At. Data Nucl. Data Tables, 23, 63-81, 1979.

Lean, J. L., and A. Skumanich, Variability of the Lyman alpha flux with solar activity, J. Geophys. Res., 88, 5751-5759, 1983.

Lysak, R. L., and C. T. Dum, Dynamics of magnetosphere-ionosphere coupling including turbulent transport, J. Geophys. Res., 88, 365380, 1983.

McFarland, M., D. L. Albritton, F. C. Fehsenfeld, E. E. Ferguson, and A. L. Schmeltekopf, Flow-drift technique for ion mobility and ionmolecule reaction rate constant measurements, 2, Positive ion reactions of $\mathrm{N}^{+}, \mathrm{O}^{+}$, and $\mathrm{N}_{2}^{+}$with $\mathrm{O}_{2}$ and $\mathrm{O}^{+}$with $\mathrm{N}_{2}$ from thermal to $2 \mathrm{eV}$, J. Chem. Phys., 59, 6620-6628, 1973.

Mehta, N. C., Ionospheric electrodynamics and its coupling to the magnetosphere, Ph.D. thesis, Univ. of Calif., San Diego, 1978.

Ogawa, T., and T. Shimazaki, Diurnal variations of odd nitrogen and ionic densities in the mesosphere and lower thermosphere: Simultaneous solution of photochemical-diffusive equations, $J$. Geophys. Res., 80, 3945-3960, 1975.

Rasmussen, C. E., and R. W. Schunk, Ionospheric convection driven by NBZ currents, J. Geophys. Res., 92, 4491-4504, 1987.

Rasmussen, C. E., R. W. Schunk, J. J. Sojka, V. B. Wickwar, O. de la Beaujardiere, J. Foster, J. Holt, D. S. Evans, and E. Nielsen, Comparison of simultaneous Chatanika and Millstone Hill observations with ionospheric model predictions, J. Geophys. Res., 91, 6986-6998, 1986.

Rees, M. H., Auroral ionization and excitation by incident energetic electrons, Planet. Space Sci., 11, 1209-1218, 1963.

Reiff, P. H., Models of auroral-zone conductances, in Magnetospheric Currents Geophys. Monogr. Ser., vol. 28, AGU, Washington, D.C., 1983.

Roble, R. G., R. E. Dickinson, and E. C. Ridley, Global circulation and temperature structure of thermosphere with high-latitude plasma convection, J. Geophys. Res., 87, 1599-1614, 1982.

Rowe, J. F., and J. D. Mathews, Low-latitude nighttime $E$ region conductivities, J. Geophys. Res., 78, 7461-7470, 1973.

Schunk, R. W., Mathematical structure of transport equations for multispecies flows, Rev. Geophys., 15, 429-445, 1977.

Schunk, R. W., and A. F. Nagy, Ionospheres of the terrestrial planets, Rev. Geophys., 18, 813-852, 1980.

Schunk, R. W., and W. J. Raitt, Atomic nitrogen and oxygen ions in the daytime high-latitude $F$ region, $J$. Geophys. Res., 85, 1255-1272, 1980.

Schunk, R. W., and J. C. G. Walker, Theroetical ion densities in the lower ionosphere, Planet. Space Sci., 21, 1875-1896, 1973.

Schunk, R. W., W. J. Raitt, and P. M. Banks, Effect of electric fields on 
the daytime high-latitude $E$ and $F$ regions, J. Geophys. Res., 80, 3121-3130, 1975.

Smith, F. L., and C. Smith, Numerical evaluation of Chapman's grazing incidence integral $\operatorname{ch}(X, \chi), J$. Geophys. Res., 77, 3592-3597, 1972.

Sojka, J. J., W. J. Raitt, and R. W. Schunk, A theoretical study of the high-latitude winter $F$ region at solar minimum for low magnetic activity, J. Geophys. Res., 86, 609-621, 1981.

Spiro, R. W., P. H. Reiff, and L. J. Maher, Jr., Precipitating electron energy flux and auroral zone conductances: An empirical model, $J$. Geophys. Res., 87, 8215-8227, 1982.

St.-Maurice, J.-P., and R. W. Schunk, Ion-neutral momentum coupling near discrete high-latitude ionospheric features, J. Geophys. Res., 86, 11,299-11,321, 1981.

Strobel, D. F., C. B. Opal, and R. R. Meier, Photoionization rates in the night-time $E$ - and $F$-region ionosphere, Planet. Space Sci., 28, 1027 $1033,1980$.

Torr, D. G., M. R. Torr, H. C. Brinton, L. H. Brace, N. W. Spencer, A. E. Hedin, W. B. Hanson, J. H. Hoffman, A. O. Nier, J. C. G. Walker, and D. W. Rusch, An experimental and theoretical study of the mean diurnal variation of $\mathrm{O}^{+}, \mathrm{NO}^{+}, \mathrm{O}_{2}^{+}$, and $\mathrm{N}_{2}^{+}$ions in the mid-latitude $F_{1}$ layer of the ionosphere, J. Geophys. Res., 84, 33603372, 1979.
Torr, M. R., and D. G. Torr, Ionization frequencies for solar cycle 21: Revised, J. Geophys. Res., 90, 6675-6678, 1985.

Vickrey, J. F., R. R. Vondrak, and S. J. Matthews, The diurnal and latitudinal variation of auroral zone ionospheric conductivity, $J$. Geophys. Res., 86, 65-75, 1981.

Vondrak, R., and R. Robinson, Inference of high-latitude ionization and conductivity from AE-C measurements of auroral electron fluxes, J. Geophys. Res., 90, 7505-7512, 1985.

Wallis, D. D., and E. E. Budzinski, Empirical models of height integrated conductivities, J. Geophys. Res., 86, 125-137, 1981.

Watanabe, K., F. M. Matsunaga, and H. Sakai, Absorption coefficient and photoionization yield of NO in the region 580-1350 \&, Appl. Opt., 6, 391-396, 1967.

C. E. Rasmussen and R. W. Schunk, CASS, Utah State University, UMC 4405, Logan, UT 84322.

V. B. Wickwar, SRI International, 333 Ravenswood Avenue, Menlo Park, CA 94025.

(Received August 17, 1987;

revised March 29, 1988;

accepted March 30, 1988.) 